\title{
Uma espécie única de Maprounea (Euphorbiaceae) na América do Sul
}

\author{
Antonio Costa Allem (")
}

\section{Resumo}

O autor mostra que Maprounea guyanensis Aublet e Maprounea brasiliensis St. Hil. (Euphorbiaceae) constituem uma única espécie, de ampla dispersão geográfica.

\section{INTRODUÇÃo}

O gênero Maprounea foi descrito por Aublet em 1775, constituindo-se àquela época em gênero monotípico. A única espécie a integrálo era $M$. guyanensis Aublet, com distribuição citada para a Amazônia e Guianas.

Saint Hilaire (1824) descreveu M. brasiliensis; Müller Argoviensis (1866) descreveu M. africana (África) e Pax (1912) descreveu M. membranacea (África). Assim, o gênero Maprounea ficou constituído por quatro espé cies, duas africanas e duas americanas.

$\mathrm{O}$ autor deste trabalho teve a oportunidade de examinar Maprounea em campo, no Estacoo de Goiás e no Distrito Federal, além de dispor para exame de farto material depositado no herbário do Departamento de Botânica da Universidade de Brasília (UB). Estas felizes circunstâncias levaram-no à conclusão de que $M$. guyanensis e $M$. brasiliensis representam una única entidade taxonômica.

\section{Maprounea Aublet}

Hist. Pl, Guyan, 2:895.t.342. 1775; Saint-Hilaire, Pl. Us, Brés. t. 65 1824; Müll. Arg. in D.C. Prodr. 15(2) : 1190.1866 et in Martius, Fl. Bras. 11(2): 541.t.81.1874; Pax in Engler, Das Pflanzenreich IV. 147-V (Heft 52) : 175. figs. 32-33. 1912.

Maprounea guyanensis Aublet, Hist. PI. Guyan. 2:895. t. 342.1775.

M. brasiliensis St. Hil., PI. Us. Brés t. 65.1824 , syn. nov.

Nome popular: Vaquinha (Estado de Minas Gerais); Marmeleiro-do-campo (segundo Saint Hilaire).
Saint Hilaire (1824) descreveu a espécie com base em indivíduos coletados no Estado de Minas Gerais (Porto de Quebra Anzol e em Minas Novas). A região de Minas Novas é de cerrado e, neste ambiente, Maprounea, geralmente, apresenta-se com hábito subarbustivo ou arbustivo. Saint Hilaire (1824) descreve a espécie, dizendo ser ela "sous-arbrisseau de 2 à 3 pieds". Este hábito é condicionado por fa. tores ecológicos.

Müller Argoviensis (1874:542) separa as duas espécies aqui abordadas da seguinte maneira:

- Bracteae masc. rhombeae subulato- acuminatae, capsulae tridymae majusculae -1 . M. brasiliensis.

- Bracteae masc. latae exiguae brevissime acutatae, capsulae globosae parvulae -2 . M. guyanensis.

Müller Argoviensis (1874) diz, na chave, que $M$. brasiliensis tem "bracteae masc. rhombeae subulato-acuminatae", mas na descriação da espécie (ibid.: 542) relata "bracteis masculis e basilate ovata $v$. obovata subulato-acuminatis". O desenho da bráctes masculina (ibid.: fig. 81) mostra uma estrutura que combina com a descrição do texto. M. brasiliensis tem, segundo Müller Argoviensis (1874), "capsulae tridymae majusculae", enquanto $M$. guyanensis tem "capsulae gio. bosae parvulae". Entretanto, o próprịo Müller Argoviensis se contradiz, pois na descriçãc de M. guyanensis (ibid.: 544) relata: "Capsulae 4. $\mathrm{mm}$ longae, $5 \mathrm{~mm}$ latae, leviuscule tridymae". Esta descrição não difere em nada da que ele atribuiu a $M$. brasiliensis (ibid.: 542): "capsulic majusculis (10 mm long.) leviuscule tridymis".

Deduz-se que a chave dicotômica elaboreda por Müller Argoviensis é extremamente ar tificial e carente de maior valor taxonômico A observação de indivíduos de Maprounea, et campo, no Estado de Goiás e no Distrito Fede ral mostrou que as cápsulas são geralment

(*) - Centro Nacional de Recursos Genéticos (CENARgen/EMBrAPA). Brasilia, D.F. 
globosas, mas que, às vezes, os carpelos apresentam o dorso muito levemente carenado, devido a uma elevação da linha de sutura, que une as duas valvas que constituem o carpelo.

Pax (1910: 175) também parece ter perce bido este detalhe, pois, em sua chave, omitiu qualquer aspecto referente ao fruto:

a. Bracteae ồ rhombeae, subulato-acuminatae - 1. M. brasiliensis.

b. Bracteae of latae, exiguae, breviter acutae - 2. M. guyanensis.

Percebe-se pelo exame da chave de Pax, que este autor também não conseguiu encontrar diferença significativa entre as duas espé. cies, e, numa atitude conservadorista. preferiu seguir o pensamento de Müller Argoviensis (1874). Pax, em sua chave, diz que M. brasiliensis tem brácteas masculinas "rhombeae, subulato-acuminatae" e, na descrição da espécie, relata "bracteae of ovatae, acuminatae" (ibid.: 176). De M. guyanensis ele diz, na chave, que tem brácteas masculinas "latae, exiguae, breviter acutae", e na descrição da espécie relata "bracteae utriusque sexus latissimae ovatae, exiguae, brevissimae acutatae" (ibid.: 177).

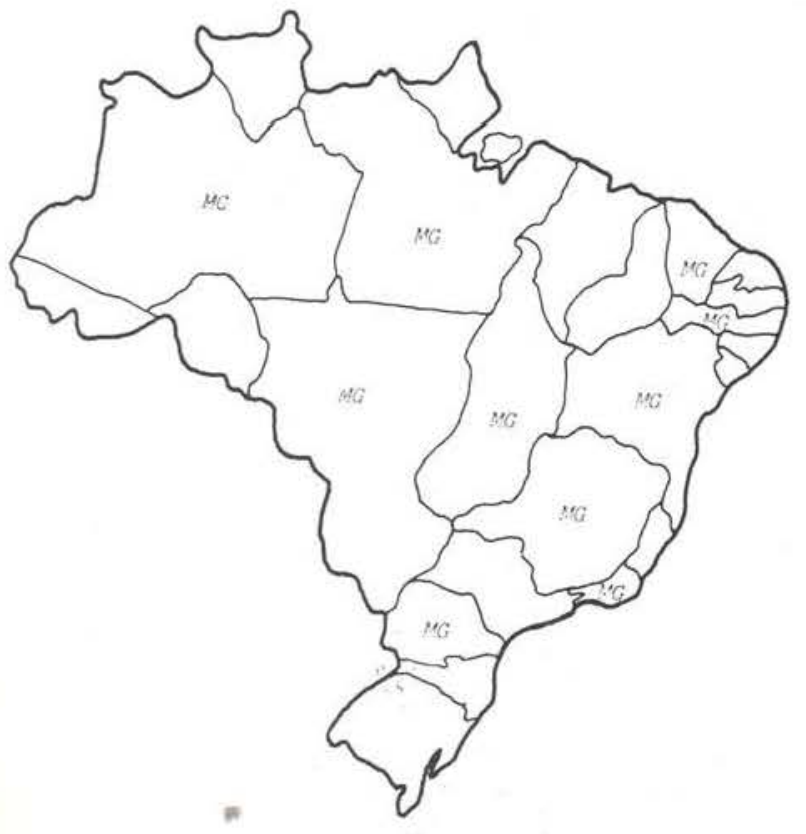

Fig. 1 - Distribuição conhecida de Maprounea guyanensis (MG) no Brasil.
Não há oposição maior entre uma e outra diagnose, na tentativa de separação das duas espécies. É completamente inexpressivo pensar-se na possibilidade de separar duas espé cies dum gênero com base apenas na morfologia das brácteas florais. Isto é válido para todas as espécies de Euphorbiaceae. A morfologia das brácteas florais, assim como de estípulas e folhas, é geralmente variável.

Outro aspecto importante é o da cistribuição geográfica atribuída às duas espécies. Consultando Müller Argoviensis (1874) e Pax (1912), temos o seguinte quadro:

M. brasiliensis M. guyanensis

\begin{tabular}{lll} 
BRASIL & & \\
Mato Grosso & $\mathrm{X}$ & $\mathrm{X}$ \\
Minas Gerais & $\mathrm{X}$ & $\mathrm{X}$ \\
Bahia & $\mathrm{X}$ & $\mathrm{X}$ \\
Goiás & & $\mathrm{X}$ \\
Ceará & & $\mathrm{X}$ \\
Amazonas & $\mathrm{X}$ & $\mathrm{X}$ \\
Pará & & $\mathrm{X}$ \\
Rio de Janeiro & & $\mathrm{X}$ \\
\hline Guiana Francesa & & $\mathrm{X}$ \\
Guiana Holandesa (Suriname) & $\mathrm{X}$ \\
Guiana Inglesa & & $\mathrm{X}$ \\
Colômbia & $\mathrm{X}$ \\
Peru & & $\mathrm{X}$ \\
Bollvia & &
\end{tabular}

E relevante destacar que as duas espécies se superpõem nos Estados brasileiros de Mınas Gerais, bahia, Goiás e Kio de Janeıro, segundo a distribuiçăo relatada por Pax e Müller Argoviensıs. E surpreendente que nenhum autor tenha percebido estas evidências, as quaıs, sumadas às características distintas interespecificas inexpressivas já debatidas, demonstram estar-se diante duma única espécie, com grande raio de dispersão.

Hatschbach \& Fernandes (1964) relatam a presença de $M$. brasiliensis no Estado do Paraná. Este parece ser o limite austral da espé. cie. A distribuição geográfica conhecida de M. guyanensis está documentada nas Figuras 1 e 2 . 


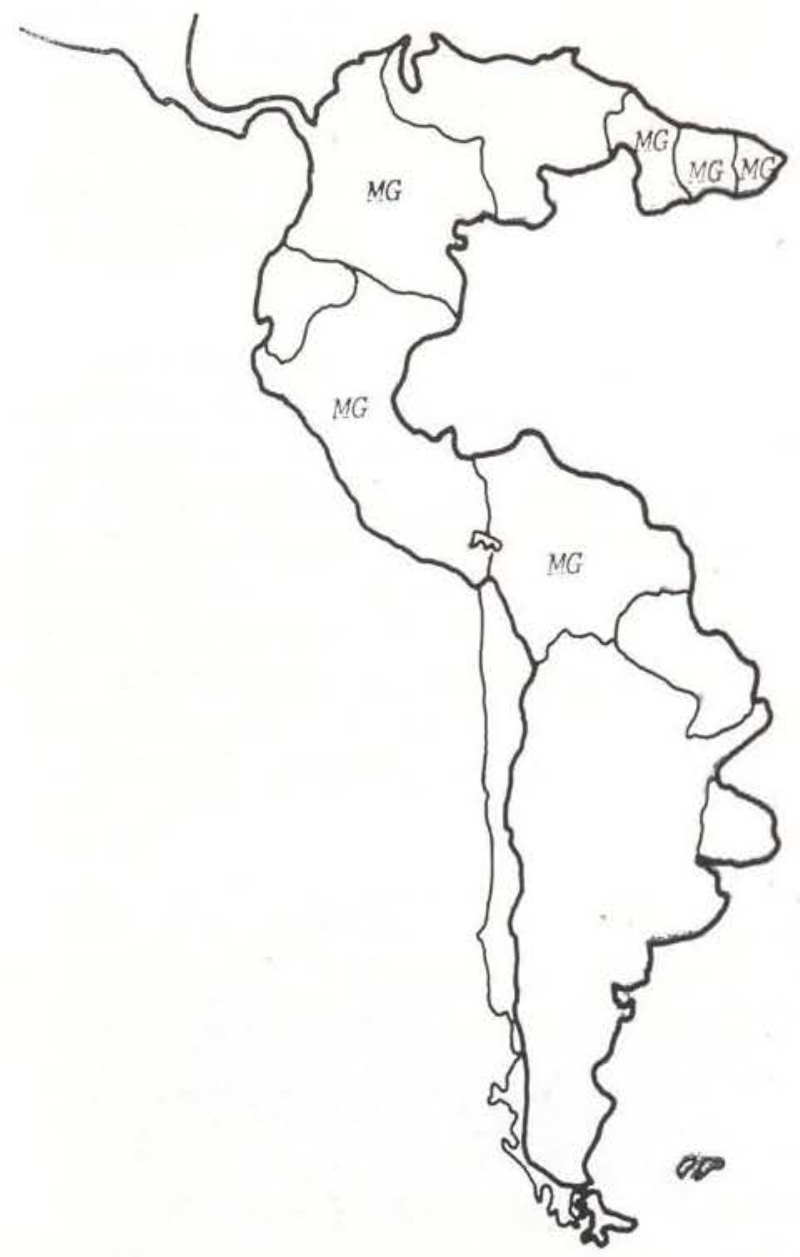

Fig. 2 - Distribuição conhecida de Maprounea guyanensis (MG) na América do Sul.

\section{ConClusões}

1) M. guyanensis é uma espécie bastante variável em características vegetativas, sendo as características florais e do fruto mais estáveis.

$\mathrm{Na}$ região dos cerrados (savanas) brasileiros, esta espécie apresenta-se freqüentemente com o hábito subarbustivo ou arbustivo (0,5-1,5 $\mathrm{m}$ de altura). Este hábito está representado geralmente em locais onde não há água em quantidade apreciável na superfície do solo, em campos cerrados ou em campos grossos. Na mesma região, basta a presença de um córrego, caracteri- zando uma mata ciliar ou de galeria, e já enconiramos arvoretas de 3,4 ou 5 metros de altura. O diâmetro do caule dum subarbusto ou arbusto oscila em torno de $1,3 \mathrm{~cm}$. enquanto o duma arvoreta oscila em torno de $4-7 \mathrm{~cm}$. É interessante registrar que mesmo subarbustos de 0,5-1 $\mathrm{m}$ de al'ura são encontrados com flores e frutos. As espigas masculinas, estrobiliformes, são deciduas, e, muitas vezes, encontram-se, somente frutos jovens ou maduros na planta. Para o menos avisado, parece estar-se diante duma espécie dióica. A espécie ocorre também em florestas, à beira da mata. Nestas condições ecológicas, a espécie apresenta um hábito bastante dis tinto; Hatschbach \& Fernandes (1964) relatam que o material por eles examinado - Paraná, Município de Guaratuba, Morro de Morretes, mata pluvial, 17.XII.1963. Hatschbach 10779 - proveio de uma árvore de $18 \mathrm{~m}$ de altura. A espécie apresenta folhas de morfologia variável (subelípticas, lanceoladas, ovaladas etc), oscilando a consistência desde membranáceas até coriáceas (em seco). A venação foliar também é variável. As dimensões da folha são variáveis, porém, raramente, ultrapassam $7-8 \mathrm{~cm}$ de comprimento, Em seco, e mesmo em fresco, as folhas, freqüentemente, são brilhantes na face superior, isto provavelmente devido à existência duma camada de cera, recobrindo a epiderme superior.

2) Há autores que poderiam interpretar a situação exposta neste trabalho sob o aspecto de vicariância. Assim, os indivíduos ocorrentes em cerrado seriam uma espécie, e aqueles ocorrentes em mata seriam uma outra espécie, constituindo espécies vicariantes, cuja maior diferença residiria em características vegetativas (consistência, brilho e morfologia foliar) e no hábito (subarbusto ou arbusto $X$ árvore). O pensamento de que "grupos naturais bem definidos" devam ser considerados gêneros ou espécies distintas repousa em hábito e morfologia de caracteres vegetativos, impcrtantes na adaptação ao ambiente, sem atentar para outros aspec. tos menos influenciáveis pelo ambiente, 
tais como a morfologia floral e do fruto. além dos grãos de pólen. Esse pensamento deve ser severamente criticado, pois abre um perigoso precedente na taxonomia das Angiospermas. Um taxon é, por natureza, qualquer unidade dentro do mundo dos seres vivos, em que vários indivíduos propiciam uma descrição geral que os distingue de outros relacionados. $\mathrm{Se}$ a morfologia floral e do fruto é igual, características vegetativas e hábito somente servirão para deiimitar categorias infraespecíficas. Para o caso aqui exposto, o conceito de ecótipo deve ser firmado. Os ecótipos duma espécie, em especial se estiverem isolados geograficamente, apresentarão, provavelmente, claras diferenças genotipicas, as quais poderão ser expressas através da utilização de processos moleculares (eletroforese de enzimas ou cromatografia de flavonas), porém essa condição não é obrigatória. É

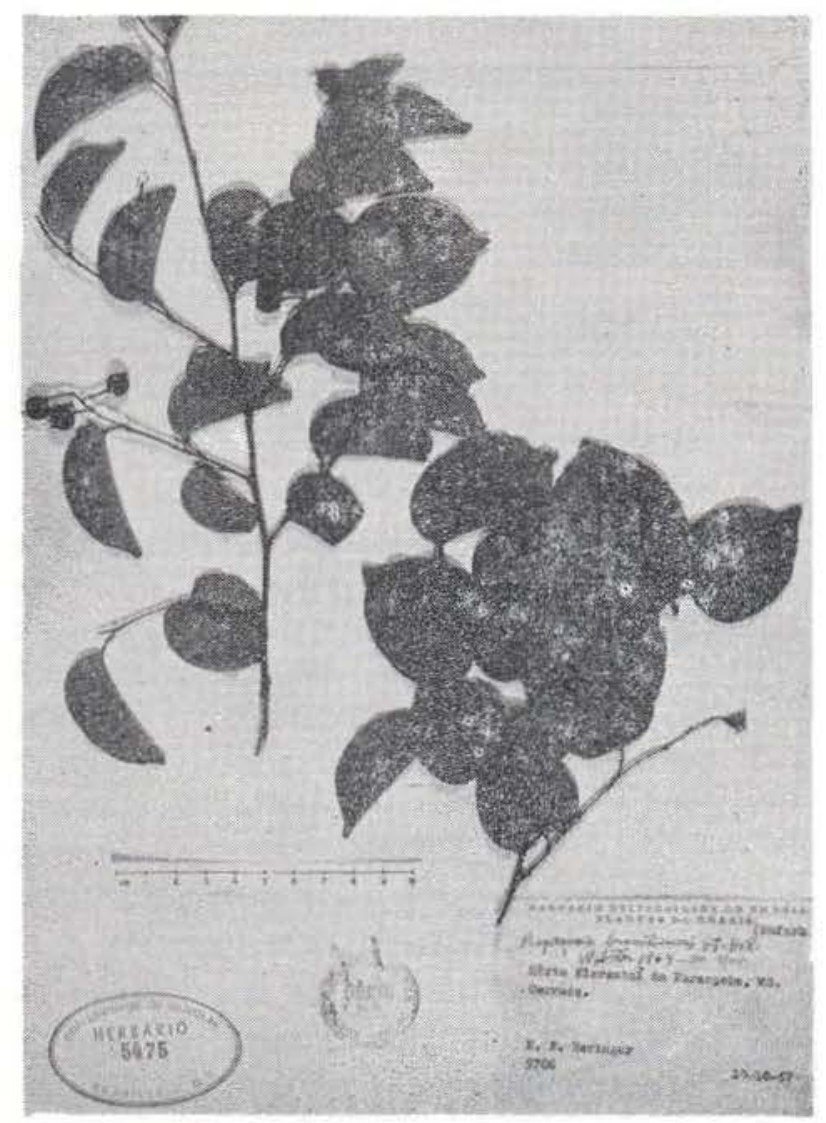

plenamente possível a existência de ecótipos que desfrutem do mesmo genótipo. e cujas diferenças fenotípicas sejam obra exclusivamente do ambiente. Visanao a fornecer dados mais completos a este estudo, correlacionou-se, sempre que possivel, característicás de hábito com características ambientais. Estes registros aparecem na relaçăo de material estudado, ao final deste trabaiho.

3) M. guyanensis parece ser espécie perenifólia, florescendo e frutificando no Estado de Goiás e no Distrito Federal desde setembro até março. As evidências indicam polinização entomófila para a espécie, dada a grande quartidade de formigas circulando sobre as flores, atraídas pela secreção das glândulas das peças florais. Há um registro de que os frutos são muito apreciados pelos pássaros (Manaus Amazonas) .

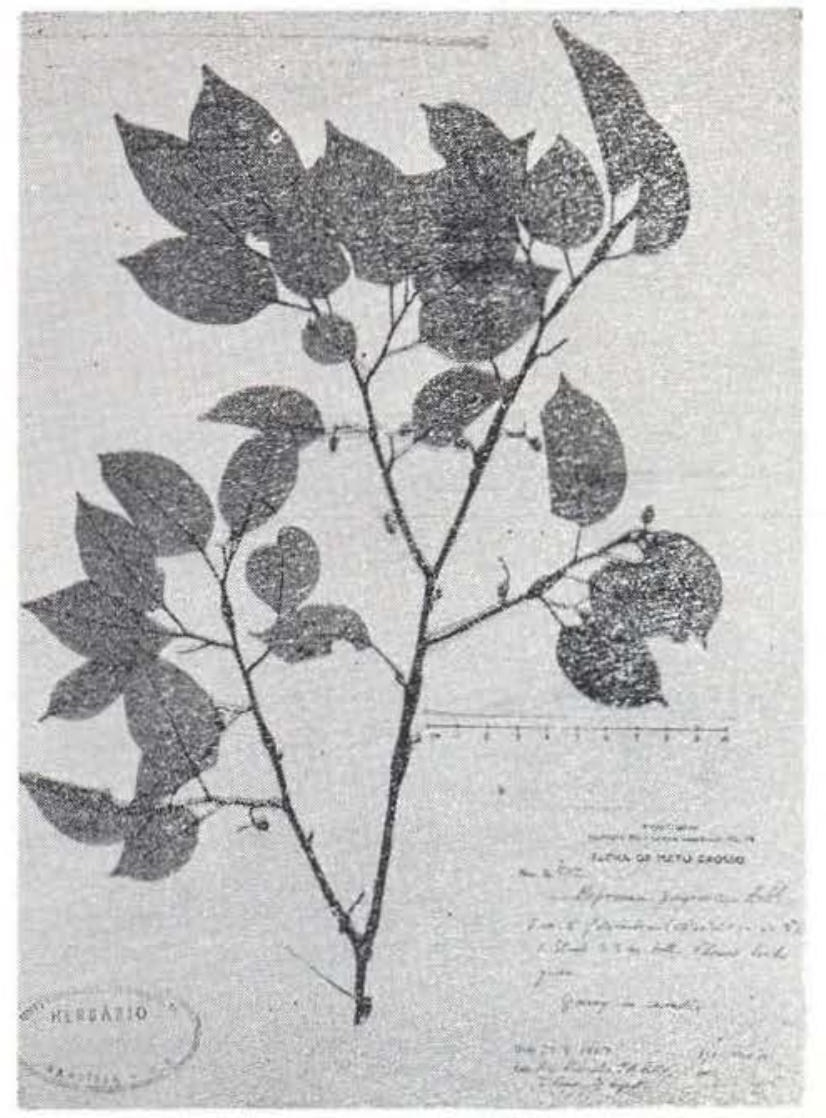

Fig. 3 - Indivíduos de Maprounea guyanensis com folhas estruturalmente distintas. A coleção E. P. Heringer 5786 apresenta folhas coriáceas, enquanto que a coleção P. W. Richards et al. apresenta folhas membranáceas. 
4.) M. guyanensis é relativamente abundante na composição da flora dos cerrados br. sileiros. Nos campos grossos e campo. cerrados circundantes a Brasília, a freqüência da espécie é elevada. Em sua dis tribuição geral, documentada, atinge os Estados brasileiros de Paraná, Rio de Janeiro, Minas Gerais, Bahia, Goiás, Distrito Federal, Mato Grosso, Pará, Amazonas, Ceará, Pernambuco. Deve ocorrer também em São Paulo. Na América do Sul, distribui-se pelas Guianas e Suriname, além da Colômbia, Peru e Bolivia. Sua ocorrência no Caribe ainda é incerta. Pax (1912: 178) relaciona em sua monografia Maprounea membranacea Pax \& Hoffm espécie ocorrente na África. O grau de parentesco desta espécie com M. guyanensis é tão evidente que $\operatorname{Pax}(1912: 178)$ escreveu uma nota a respeito de $M$. membranacea: "Species hic descripta similis et affinis est $M$. guyanensi americanae, sed foliis tenuiter membranaceis et fioribus dioicis facile recognoscitur; semına non foveolata, sed irregulariter foveolato - rugulosa". De rigor, somente a característica dióica separaria $M$. membranacea de $M$. guyanensis. O desenho de M. membranacea (ibid.: fig. 33) mostra uma pıanta muito semelhante a $M$. guyanensis. Seria interessante que, para o futuro, algum pesquisador pudesse comparar material americano e africano, a fim de poder con. frontar o real grau de separação entre estas duas espécies.

Material estudado de Maprounea guyanensis BRASIL: MINAS GERAIS - Lagoo Santa, 02. Vill.1959, E.P. Heringer 7118 (UB) Paracatu, arbusto em cerrado, 03.VI.1960. E. P. Heringer 7585 (UB) : subarbusto $1 \mathrm{~m}$ em cerrado, 05.II.1970, H. S. Irwin 26101 et al. (UB, NY) - Belo Horizonte, Serra do Taquaril, arbusto $80 \mathrm{~cm}$ em cerrado, 27.IX. 1942, J. E. Oliveira 1123 (UB) - Lagoa Santa, Horto Florestal de Paraopeba, 10.X.1957, E. P. Herınger 5786 (UB) - Conceição do Mato Dentro, Serra do Cipó, 24. VIII. 1957, E. P. Heringer 5728 (UB) $=$ - Diamantina, arbusto $2 \mathrm{~m}$ em cerrado, 16. III. 1970, H.S. Irwin 27704 et al. (UB, NY) - Sáo João da Chapada, subarbusto $75 \mathrm{~cm}$ em cerrado, 24.III.1970, H. S. Irwin 28229 et al.
(UB, NY) - Datas, arbusto $1 \mathrm{~m}$ em campos grossos, 24.I.1969. H. S. Irwin 22560 et al. (UB, NY) - Montes Claros, arbusto $1,5 \mathrm{~m} \mathrm{em}$ cerrado, 23.II.1969, H. S. Irwin 23728 et al. (UB, NY) - Patrocínio, arbusto $1 \mathrm{~m} \mathrm{em}$ campos grossos, 28.I.1970, H.S. Irwin 25507 et al. (UB;NY) - Gouveia, arbusto $1 \mathrm{~m}$ em cerrado, 03.1I.1972, Anderson, Stieber \& Kirkbride 35277 (UB,NY); arbusto $1 \mathrm{~m}$ em mata ciliar, 10.IV. 1973, W. Anderson 8545 (UB,NY) - João Piu.ieiro, 28 VII.1961, E.P. Heringer 8547 (UB) - Juiz de Fora, Coronel Pacheco, Estação Experimental de Água Limpa, árvore de $20 \mathrm{~m} \times 30$ $\mathrm{cm}$ em mata de galeria, 08.V.1969, Vascos Gomes s/n. (UB) - GOIÁS - Luziânia, entre Gama e rio Corumbá, árvore $2,5 \mathrm{~m} \times 3 \mathrm{~cm}, 19 . \mathrm{X}$. 1963, J.M. Pires 57141 (UB); Granja Samambaia, 01.I.1967, E.P. Heringer 11247 (UB) Veadeiros, Chapada dos Veadeiros, subarbusto $1 \mathrm{~m}$ em cerrado, 13.II.1966, H.S. Irwin 12737 et al. (UB,NY); Chapada dos Veadeiros, arbusto $1 \mathrm{~m}, 18$ VII. 1964, G. Prance \& N. Silva 58190 (UB, NY) - Cristalina, árvore $3 \mathrm{~m} \times 8 \mathrm{~cm}$, 03.XI.1965, H.S. Irwin 9843 et al. (UB, NY) Caiapônia, Serra do Caiapó, floresta, árvore $15 \mathrm{~m} \times 10 \mathrm{~cm}, 22 . X .1964$, G. Prance \& N. Silva 59555 (UB, NY) - Pirenópolis, Serra dos Pireneus, arvoreta $3 \mathrm{~m} \times 5 \mathrm{~cm}$ em cerrado, 17.I. 1972, H.S. Irwin 34451 et al. (UB, NY); subarbusto $75 \mathrm{~cm}$ em cerrado, 17.I.1972. H.S. Irwin 34445 et al. (UB, NY) - Catalão, árvore $3 \mathrm{~m} \times 6 \mathrm{~cm}$ em cerrado, 22.1.1970, H.S. Irwin 25155 et al. (UB, NY) - Corumbá de Goiás, árvore $6 \mathrm{~m}$ em cerrado, 14.V.1973, W. Anderson 10218 (UB, NY); Vale do rio Corumbá, árvore $3 \mathrm{~m}, 14.1 .1968$, Irwin, Maxwell \& Wasshau sen 18580 (UB, NY); Vale do rio Corumbá, vore $3 \mathrm{~m}, 14.1 .1968$, Irwin, Maxwell \& Wasshau\& Wasshausen 18794 (UB, NY) - Cabeceiras, arbusto $1 \mathrm{~m}$ em cerrado, 19.XI.1965, H.S. Irwin 10559 et al. (UB,NY). DISTRITO FEDERAL - Taguatinga, arbusto $1 \mathrm{~m}$ em cerrado, 25.XI. 1965, H.S. Irwin 10641 et al. (UB,NY) - Brasilia, subarbusto $80 \mathrm{~cm}$ em cerrado, 06.IX.1963, Cobra \& Oliveira 158 (UB); Country Club, subarbusto $50 \mathrm{~cm}, 08 . \mathrm{VI} .1965$, D. Sucre 697 (UB); Chapada da Contagem, 13.VIII.1964, irwin \& Soderstrom 5069 (UB,NY); Campus da UnB, 16.IV.1963, J.M. Pires 9121 et al. (UB); Chapada da Contagem, arbusto $2 \mathrm{~m}$ em cerrado, 04.IX.1965, H.S. Irwin 7970 et al. (UB,NY); 
Chapada da Contagem, arbusto $3 \mathrm{~m}, 25$. VIII. 1965, H.S. Irwin 7885 et al. (UB,NY): Fazenda Vargem Bonita, 19.VII.1966, D. Hunt \& J. Ramos 6687 (UB, K); 10 km E lago Paranoá, árvore $8 \mathrm{~m} \times 18 \mathrm{~cm}$ em mata ciliar, 23.II.1970, H.S. Irwin 26551 et al. (UB,NY); Escola Agrotécni$\mathrm{ca}$, árvore $10 \mathrm{~m} \times 3 \mathrm{~cm}$ à margem do córrego, 23. VII. 1963, E.P. Heringer 9155 (UB); plano piloto, 21.X.1962, E.P. Heringer 9024 (UP) - Planaltina, Colégio Agrícola, árvore $8 \mathrm{~m}$ em mata ciliar, 22.IX.1969, E.P. Heringer 11892 (UB); Córrego Monteiro, árvore $4 \mathrm{~m} \times 7 \mathrm{~cm}$ em mata ciliar, 29.IX.1965, H.S. Irwin 8777 et al. (UB, NY) - Gama, Parque Municipal do Gama, árvore $6 \mathrm{~m} \times 7 \mathrm{~cm}$ em mata ciliar, 31. YIII.1964, Irwin \& Soderstrom 5817 (UB, NY); Parque Municipal do Gama, árvore $4 \mathrm{~m} \times 7 \mathrm{~cm}$ em mata ciliar, 04.IX.1964, Irwin \& Soderstrom 5955 (UB, NY) - RIO DE JANEIRO - Rio de Janeiro, perto do Horto Florestal, árvore pequena, 14.1.1932, Paulino \& Vitório $\mathbf{s} / \mathbf{n} .^{\circ}$ (UB, RB); Vista Chinesa, árvore 6-7 m em mata, 27. XII. 1927. Sine leg. (UB, RB). BAHIA - Seabra, em direção a Água de Rega, rio Riachão, árvore $5 \mathrm{~m} \times 6 \mathrm{~cm}$ em mata, 23.11.1971, H.S. Irwin 30792 et al. (UB, NY); bancos arenosos e rochosos do rio Riachão, 26.II.1971, H.S. Irwin 31146 et al. (UB, NY); mata ciliar, 25.II.1971. H.S. Irwin 30995 et al. (UB, NY) - Morro do Chapéu, rio Ferro Doido, árvore $5 \mathrm{~m} \times 8 \mathrm{~cm}$ em mata ciliar, 19.II.1971, H.S. Irwin 32607 et al. (UB, NY). MATO GROSSO - Xavantina, arbusto $2-3 \mathrm{~m}$ em cerradão, 27.VIII.1967, Richards, Ratter \& Ramos 502 (UB); rodovia XavantinaCachimbo, arbusto $1 \mathrm{~m}$ em cerrado, 14.XI.1967, Philcox, Ramos \& Souza 3021 (UB, K); árvore $3 \mathrm{~m}$ em cerrado, 19.XII.1967, Philcox, Ferreira \& Bertoldo 3595 (UB, K); rodovia XavantinaCachimbo, arbusto $2 \mathrm{~m}$ em capoeira, 08.X.1968, Sidney \& Onishi 479 (UB); rodovia XavantinaCachimbo, 14.IX.1968, R. Harley \& R. Souza 10027 (UB, K) ; rodovia Xavantina-Cachimbo, árvore $5 \mathrm{~m}$ em beira de mata, 14.IX.1968, R. Harley \& R. Souza 10026 (UB, K) - Serra do Roncador árvore $6 \mathrm{~m} \times 5 \mathrm{~cm}$ em mata 29.IX.1964, Prance, Silva \& Murça Pires 59158 (UB) - PERNAMBUCO - São José do Belmonte, arbusto 2.5 m, 13.V.1975, D. Andrade Lima et al. 729 (UB) - PARÁ - Belém, IX.1961, J. Murça Pires 51745 (UB, NY) - AMAZONAS - Manaus, km 19 estrada do Aleixo, árvore $5 \mathrm{~m}$ em mata 30.III.1967, Prance, Pena \& Ramos 4719 (UB, NY, INPA); Campos Sales, margem do igarapé do Buião, 28.IX.1954, Chagas s/n. (UB, MG, INPA); estrada do Vai-quem-quer, 14.X.1955. Chagas $\mathbf{s} / \mathrm{n} .^{\circ}$ (UB, MG, INPA); estrada do Alei xo, 02.XII.1955, Dionísio s/n. (UB, MG, INPA): margem do igarapé do Passarinho, 17.XI.1955, Dionísio $s / n .{ }^{\circ}$ (UB, MG, INPA); igarapé da Cachoeira Baixa do Tarumä, 02.IX.1955, Francis-

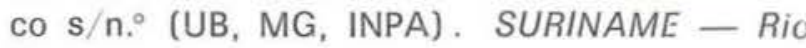
Saramacca, árvore em mata, 30.IX.1944, B. Maguire 24873 (UB, NY).

\section{SUMMARY}

The author shows that Maprounea guyanensis Aublet and Maprounea brasiliensis St. Hil. are the same species, having a wide geographical dispersion.

\section{BIBLIOGRAFIA CITADA}

Hatschbach, G. \& Fernandes, E.C.L.

1964 - Maprounea brasiliensis St. Hil. nova Euphorbiaceae para a flora paranaense. Bol. Univ. Paraná, Bot., 13 : 1-3. fig. 1-2.

MÜLLER, J.

1866 - Euphorbiaceae. In: De Candolle, Prodromus 15(2) : 189-1261.

1874 - Euphorbiaceae. In: Martius, C.F.P. Fl. Bras. 11(2) : 293-750. fig. 43-104.

PAx, F .

1912 - Euphorbiaceae-Hippomaneae. In: Engler, A. Das Pflanzenreich IV. 147.V (Heft 52) : 1-319. fig, 1-58.

Saint Hilaire, a.

1824 - Plantes usuelles des brésiliens. fig. 1-70. Paris. Imprimerie de Casimir. 